\title{
Cu cluster shell structure at elevated temperatures
}

Christensen, Ole Bøssing; Jacobsen, Karsten Wedel; Nørskov, Jens Kehlet; Manninen, M.

Published in:

Physical Review Letters

Link to article, DOI:

10.1103/PhysRevLett.66.2219

Publication date:

1991

Document Version

Publisher's PDF, also known as Version of record

Link back to DTU Orbit

Citation (APA):

Christensen, O. B., Jacobsen, K. W., Nørskov, J. K., \& Manninen, M. (1991). Cu cluster shell structure at elevated temperatures. Physical Review Letters, 66(17), 2219-2222.

https://doi.org/10.1103/PhysRevLett.66.2219

\section{General rights}

Copyright and moral rights for the publications made accessible in the public portal are retained by the authors and/or other copyright owners and it is a condition of accessing publications that users recognise and abide by the legal requirements associated with these rights.

- Users may download and print one copy of any publication from the public portal for the purpose of private study or research.

- You may not further distribute the material or use it for any profit-making activity or commercial gain

- You may freely distribute the URL identifying the publication in the public portal

If you believe that this document breaches copyright please contact us providing details, and we will remove access to the work immediately and investigate your claim. 


\title{
Cu Cluster Shell Structure at Elevated Temperatures
}

\author{
O. B. Christensen, K. W. Jacobsen, and J. K. Nørskov \\ Laboratory of Applied Physics, Technical University of Denmark, DK-2800 Lyngby, Denmark \\ M. Manninen \\ Department of Physics, University of Jyväskylä, SF-40100 Jyväskylä, Finland
}

(Received 21 December 1990)

\begin{abstract}
Equilibrium structures of small 3-29-atom $\mathrm{Cu}$ clusters are determined by simulated annealing, and finite-temperature ensembles are simulated by Monte Carlo techniques using the effective-medium theory for the energy calculation. Clusters with 8,18 , and 20 atoms are found to be particularly stable. The equilibrium geometrical structures are determined and found to be determined by a Jahn-Teller distortion, which is found to affect the geometry also at high temperatures. The "magic" clusters retain their large stability even at elevated temperatures.
\end{abstract}

PACS numbers: $68.35 . \mathrm{Bs}, 36.40 .+\mathrm{d}, 71.45 . \mathrm{Nt}$

The stability of small metal clusters is found to vary substantially with the cluster size. ${ }^{1-3}$ The stability patterns can be explained very elegantly by noting that if the stability of the cluster is plotted as a function of the number of valence electrons, the most stable clusters are found at the "magic" electron numbers 8, 18, 20, etc. This has subsequently been explained in a number of simple models, ranging from "spherical well" models known from nuclear physics to jellium-model calculations within the local-density approximation. ${ }^{4,5}$ In all these cases the atomic structure of the solid is neglected, and the electrons are assumed to move in a spherically symmetrical potential. Distorted droplets have been described by Clemenger ${ }^{6}$ in the Nilsson-oscillator model; ${ }^{7}$ recently, Ekardt and Penzar has described spheroidal jellium droplets. ${ }^{8}$

Very small clusters (up to 3-4 atoms) have been treated in "exact" configuration-interaction calculations. 9 For larger metal clusters, even approximate calculations including the geometry and electronic structure have been restricted to relatively small clusters, containing only about 10 atoms, and to the zero-temperature ground state. ${ }^{9,10}$ All these calculations show that, due to the Jahn-Teller distortion, there is an interplay between the geometry and the electronic structure in the clusters. The only rigorous treatment where the finite-temperature properties are considered is due to Ballone et al. ${ }^{11}$ who made an extensive study of the $\mathrm{Na}_{20}$ cluster.

In this Letter we present a systematic study of the stability, structure, and finite-temperature properties of all neutral $\mathrm{Cu}$ clusters with up to 29 atoms. We show that even for large clusters there is a strong coupling between the geometry and the stability of the cluster. In all cases, the most stable structure is to a large extent determined by the Jahn-Teller effect, and only for the magic numbers 8 and 20 and for the "close-packed" 13-atom cluster is the resulting structure nearly spherical. In spite of the strong coupling between structure and stabil- ity of the cluster we find that the shell structure is largely independent of the temperature (in the phonon system) of the cluster.

The results of this investigation are based on extensive Monte Carlo simulations. Such simulations are extremely tedious using ab initio methods to calculate the total energy of the system, and are at present not possible at all for $\mathrm{Cu}$ clusters. We have therefore chosen to use an approximate total-energy method, the effective-medium theory. ${ }^{12}$ The total-energy expression is derived from density-functional theory, and has proven very reliable in simulations and structure determinations for bulk metal and metal surfaces. ${ }^{13}$ The expression for the total energy of a system of atoms is, in this method,

$$
E_{\text {tot }}=\sum_{\text {atoms }} E_{c}\left(\bar{n}_{i}\right)+\sum_{\text {atoms }} \Delta E_{\mathrm{AS}}^{(i)}+\Delta E_{1-\mathrm{el}} .
$$

The influence of the metallic surroundings on an atom is expressed as a function of the embedding density $\bar{n}$, which is the average electron density contributed by surrounding atoms in a neutral sphere around the atom. The function $E_{c}(\bar{n})$ is calculated once and for all within the local-density approximation by embedding one copper atom in a homogeneous electron gas. The atomicsphere correction $\Delta E_{\mathrm{AS}}$ describes the difference in the electrostatic interaction between the atoms in the system under consideration and in a close-packed (fcc) arrangement [where the electrostatic interactions are already included in the $E_{c}(\bar{n})$ function].

The calculation of the first two terms of Eq. (1) is described elsewhere. ${ }^{12}$ These two terms are sufficient to give a good description of bulk copper and of $\mathrm{Cu}$ surfaces. For the small clusters, however, it is important to describe the discrete nature of the one-electron spectrum in some detail. This is what enters through the last term in Eq. (1), which involves the difference in the oneelectron spectrum in the small cluster and in an fcc crys- 
tal of the metal with the same $\bar{n}$ :

$$
\Delta E_{1-\mathrm{el}}=\sum_{\mathrm{occ}} \epsilon_{i}(H)-\sum_{j} E_{1-\mathrm{el}}^{\mathrm{fcc}}\left(\bar{n}_{j}\right)
$$

We use a simple tight-binding Hamiltonian $H$ with one $(4 s)$ state per $\mathrm{Cu}$ atom to evaluate $\Delta E_{1 \text {-el. }}$. The matrix elements $H_{i j}$ between atomic orbitals $i$ and $j$ are taken to depend only on the interatomic distance $r_{i j}$. The matrix elements will be roughly proportional to the amplitude of orbital $i$ at site $j$ and vice versa. We take the radial dependence of this quantity to be exponential and proportional to $\left(\bar{n}_{i}^{(j)}\right)^{1 / 2}$, where $\bar{n}_{i}^{(j)}$ is the contribution to the background density $\bar{n}_{i}$ from atom $j$ :

$$
H_{i j}=-b e^{-\left(\eta_{2} / 2\right) r_{i j}}, i \neq j \text {. }
$$

The exponent $\eta_{2}$ originates from the approximate formula $^{12} \bar{n}_{i}^{(j)} \propto \exp \left(\eta_{2} r_{i j}\right)$. The prefactor $b$ is a real and positive constant, as we are dealing with $s$ orbitals only.

The density of states (DOS) of a tight-binding fcc lattice may be calculated easily. In the calculation $E_{\mid-e l}^{\mathrm{fcc}}$ is parametrized with a power law from a number of DOS calculations at different lattice constants. The prefactor $b$ in Eq. (3) is determined by equating the distance from the band bottom to the Fermi energy with the result published by Moruzzi, Janak, and Williams ${ }^{14}$ for the $\mathrm{Cu}$ $4 s$ band in their self-consistent band-structure calculation.

For cluster sizes between 3 and 29 we have made simulated annealing calculations with the Metropolis algorithm ${ }^{15}$ in order to find the equilibrium configuration for each size $N$. Several runs were made with a maximum number of energy calculations of $60000 \mathrm{~N}$ and starting temperatures between 100 and $1200 \mathrm{~K}$. Also, runs with $\Delta E_{1-\text { el }}=0$ were made for comparison. Furthermore, thermodynamic ensembles were simulated with the Monte Carlo method at a number of temperatures between 230 and $930 \mathrm{~K}$. This simulates the effect of heating the phonon system, while the electronic system is kept at zero temperature. Recently it has been shown ${ }^{16}$ that the electronic temperature may have important effects if there are large level degeneracies of some clusters. This introduces entropy effects that smear out magic numbers at high temperature. In the present calculation we never see large level degeneracies (cf. Fig. 2 ), and since we are primarily interested in the effects of temperature on the ionic degrees of freedom we have not considered the effect of temperature on the electron system here.

In Fig. 1 we plot the deviation of the calculated equilibrium energies from a standard "nuclear" fit ${ }^{2}$

$$
E_{\mathrm{fit}}=a N+b N^{2 / 3}+c N^{1 / 3} \text {, }
$$

where $a$ represents the bulk energy per atom, $b$ describes the surface energy, and $c$ is a curvature term. The fit includes the smooth changes in the total energy of the clusters with $N$, and the deviation shown in Fig. 1 is good for

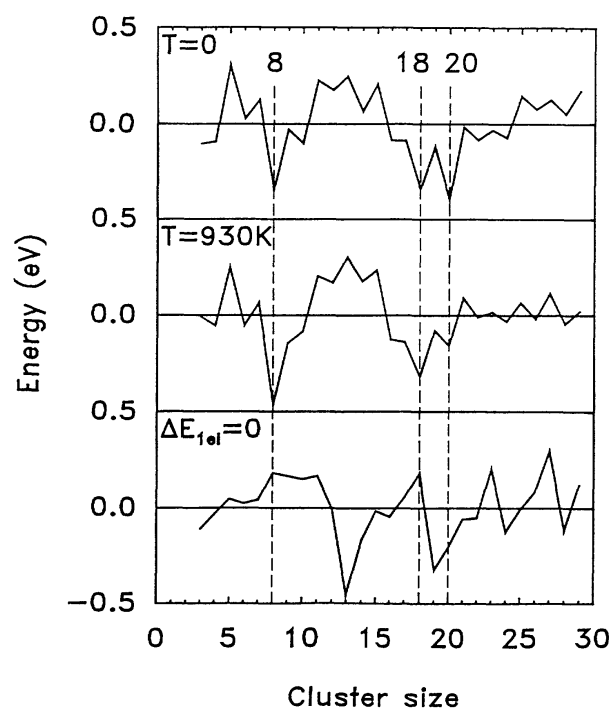

FIG. 1. Deviation of the calculated total energy $E_{\text {tot }}$ from the fit by Eq. (4). Results are shown with and without $\Delta E_{1 \text {-el }}$ from Eq. (2) included, as well as a thermal average at $T=930$ K. Each set of data has been fitted by Eq. (4) separately. The dashed lines indicate the magic numbers 8,18 , and 20 .

singling out anomalies. It is clear from the figure that the clusters of the magic sizes 8,18 , and 20 are anomalously stable in our calculation including $\Delta E_{1 \text {-el }}$ from Eq. (2). This trend is in good agreement with experiments on $\mathrm{Cu}_{N}{ }^{+}$clusters. ${ }^{17}$ Also, the calculated equilibrium structures are in good agreement with previously obtained results for alkali metals, at the sizes available. ${ }^{10,11}$ For further information on this point, see Ref. 17.

It is also seen in Fig. 1 that the magic numbers are not observed in the calculation with $\Delta E_{1-\mathrm{el}}=0$. This strongly points to the one-electron spectrum as responsible for the magic numbers, in good agreement with the simple models. In Fig. 2 we show the calculated one-electron spectra for all the cluster sizes considered. It is seen that the rather crude tight-binding model is able to reproduce a level grouping resembling spectra obtained from the selfconsistent jellium calculations. ${ }^{2,4}$ Furthermore, it is seen that all the clusters show a gap in the one-electron spectrum at the highest occupied level, and that the magic numbers are merely characterized by larger gaps than the rest. These gaps are not always present in geometries different from the equilibrium one. Calculating the total energy using the full-energy expression Eq. (1) of the structures determined from the simulated annealing with $\Delta E_{1-\mathrm{el}}=0$ does not give rise to especially stable clusters at $N=8,18$, and 20 , nor does this happen in a sequence of structures of almost-spherical chunks of a perfect fcc lattice. ${ }^{18}$ The one-electron spectra do not show gaps at the Fermi energy either. The existence of appreciable Fermi gaps of the even-sized clusters mani- 


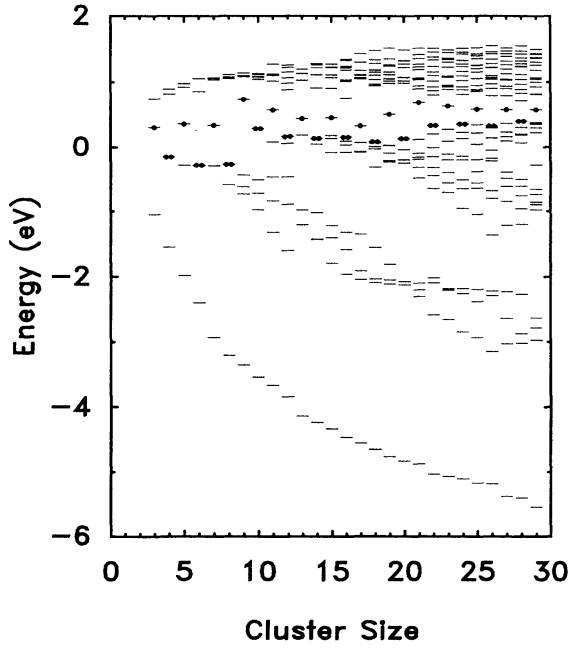

FIG. 2. One-electron levels in the calculated equilibrium structure according to Eq. (3). The highest occupied levels are indicated with solid circles (one for each electron occupying it).

fests itself in the strong even-odd oscillation of the total energy recognized in Fig. 1. A similar oscillation is not present in the two comparative calculations mentioned above. The picture emerges that the structures of the clusters are determined by a Jahn-Teller-like distortion of the system. For each system the structure distorts to maximize the gap at the Fermi level under the constraints set up by the other energy terms in Eq. (1). For even clusters there are two electrons in the highest occupied orbital and thus the energy gain due to the distortion is larger, resulting in a larger gap. For small clusters our results for the level structure are in qualitative agreement with the Hückel results of Lindsay, Wang, and George. ${ }^{19}$

The shapes of the equilibrium geometries are most conveniently illustrated by the eccentricities shown in Fig. 3. Only the magic clusters $N=8$ and 20 and the close-packed $N=13$ cluster are almost spherical; the rest resemble ellipsoids. A similar effect has been observed in the jellium-model calculations, when allowing for the possibility that the jellium background assumes a spheroidal instead of a spherical shape ${ }^{8}$ as well as in a Nilsson-oscillator model. ${ }^{6}$ The equilibrium shape of a cluster is not entirely determined by the preferences of the system of conduction electrons as described in the above-mentioned references, nor entirely by the preferences of the ion cores to close packing, as described for instance in the effective-medium theory with $\Delta E_{1-\mathrm{el}}=0$. There exists a rather even competition between the two physical effects.

It can also be seen from Fig. 1 that the magic numbers remain magic at elevated temperatures. To illustrate why, we plot the temperature-averaged density of states

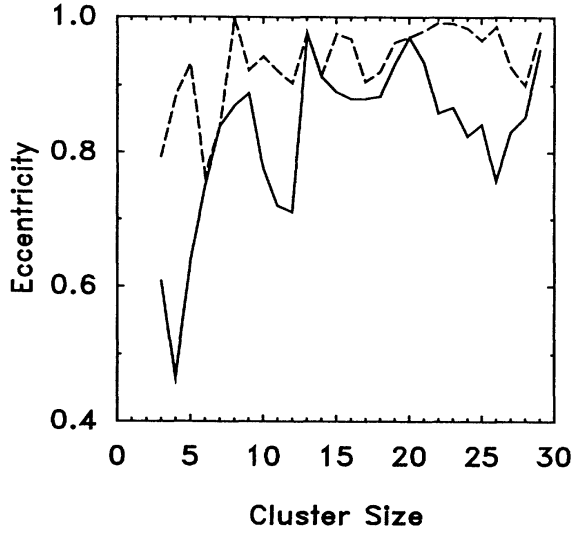

FIG. 3. Eccentricity, the square root of the ratio of the two smallest moments of inertia to the largest, for the calculated equilibrium structures.

for the magic cluster size $N=20$ at the temperature $T=930 \mathrm{~K}$ in Fig. 4. Even though there are now states at $E_{F}$ there is still a considerable dip in the DOS resulting in a lower $\Delta E_{1 \text {-el }}$ than is the case for nonmagic clusters.

The temperature $930 \mathrm{~K}$ is by no means small. It is about two-thirds of the bulk melting point of $\mathrm{Cu}$. In small clusters, which have a very large surface area, we expect a decrease in melting temperature from its bulk value. By studying the total energy and the variation of atomic structure in various clusters we do see indications of melting well below $T=930 \mathrm{~K}$. At this temperature the energy deviates profoundly from the harmonic expectation and we observe huge fluctuations in the cluster geometry. ${ }^{18}$ Thus, the finding that the clusters remain magic at high temperatures is not due to lack of mobility of the atoms. Rather, the implication is that the gap of Fig. 4 is so large that the cluster atoms will move only in

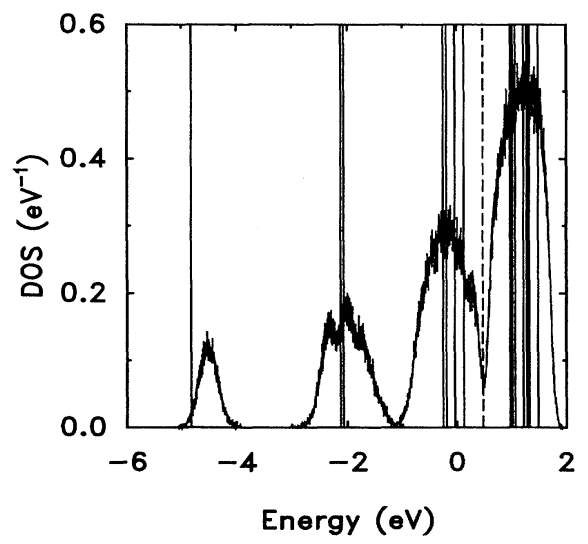

FIG. 4. Temperature-averaged density of states for the cluster $N=20$ at $T=930 \mathrm{~K}$. Dashed line: average $E_{F}$. Solid vertical lines: energy levels from equilibrium structure, i.e., the spectrum at $T=0$. The gap is still clearly to be found. 
a region of phase space where the gap remains large.

In conclusion, we have shown that the effectivemedium theory for the total energy of metal clusters correctly describes the magic numbers observed experimentally. The energy calculation is simple enough that extensive simulated annealing has been possible to determine an unbiased equilibrium geometry. We find that the geometry is closely coupled to the one-electron spectrum, and to a large extent determined by the JahnTeller effect. The special stability of the magic clusters is related to a particularly large gap at the Fermi level. Monte Carlo simulations of the finite-temperature properties show that the magic numbers remain stable. This is not because the gap in the one-electron spectrum, which is responsible for the large stability of the magic clusters, is independent of geometry. It is rather because the gap is larger than the variations of the one-electron level positions due to thermal fluctuations of the nuclear positions, at least up to $1000 \mathrm{~K}$.

Stimulating interaction with S. Bjørnholm and his group is gratefully appreciated. The research has been supported by the Danish Research Councils through the Center for Surface Reactivity.

${ }^{1}$ W. D. Knight, K. Clemenger, W. A. de Heer, W. A. Saunders, M. Y. Chou, and M. L. Cohen, Phys. Rev. Lett. 52, 2141 (1984).

${ }^{2}$ W. A. de Heer, W. D. Knight, M. Y. Chou, and M. L. Cohen, Solid State Phys. 40, 93 (1987).

${ }^{3}$ S. Bjørnholm, Contemp. Phys. 31, 309 (1990).

${ }^{4}$ W. Ekardt, Phys. Rev. B 29, 158 (1984); Surf. Sci. 152/ 153, 180 (1985).

${ }^{5}$ M. Manninen, Solid State Commun. 59, 281 (1986); M.
Manninen, R. M. Nieminen, and M. J. Puska, Phys. Rev. B 33, 4289 (1986).

${ }^{6}$ K. Clemenger, Phys. Rev. B 32, 1359 (1985).

${ }^{7}$ S. G. Nilsson, Kgl. Dan. Vidensk. Selsk. Mat. Fys. Medd. 29, No. 16 (1955); C. Gustafson, I. L. Lamm, B. Nilsson, and S. G. Nilsson, Ark. Fys. 36, 613 (1967).

${ }^{8}$ W. Ekardt and Z. Penzar, Phys. Rev. B 38, 4273 (1988).

${ }^{9}$ J. Koutećky and P. Fantucci, Chem. Rev. 86, 539 (1986), and references therein.

${ }^{10}$ J. L. Martins, J. Buttet, and R. Car, Phys. Rev. B 31, 1804 (1985); M. Manninen, Phys. Rev. B 34, 6886 (1986); J. A. Cocordan, R. Virkkunen, and M. Manninen, Phys. Scr. 38, 758 (1988); B. K. Rao, S. N. Khanna, and P. Jena, Phys. Rev. B 36, 953 (1987)

${ }^{11}$ P. Ballone, W. Andreoni, R. Car, and M. Parrinello, Europhys. Lett. 8, 73 (1989).

${ }^{12}$ K. W. Jacobsen, J. K. Nørskov, and M. J. Puska, Phys. Rev. B 35, 7423 (1987).

${ }^{13} \mathrm{~K}$. W. Jacobsen and J. K. Nørskov, in The Structure of Surfaces II, edited by J. F. van der Veen and M. A. Van Hove, Springer Series in Surface Sciences Vol. 11 (Springer, Berlin, 1988); P. Stoltze, J. K. Nørskov, and U. Landman, Phys. Rev. Lett. 61, 440 (1988).

${ }^{14}$ V. L. Moruzzi, J. F. Janak, and A. R. Williams, Calculated Electronic Properties of Metals (Pergamon, New York, 1978).

${ }^{15}$ For a review, see, for instance, Monte Carlo Methods in Statistical Physics, edited by K. Binder, Topics in Current Physics Vol. 7 (Springer, Berlin, 1979); ibid. (Springer, Berlin, 1986), 2nd ed.

${ }^{16} \mathrm{M}$. Brack, O. Genzken, and K. Hansen (to be published).

${ }^{17}$ I. Katakuse, I. Ichihara, Y. Fujita, T. Matsuo, T. Sakurai, and H. Matsuda, Int. J. Mass Spectrom. Ion Proc. 67, 229 (1985). (For the charged $\mathrm{Cu}_{N}{ }^{+}$clusters the magic numbers are 9,19 , and 21.)

${ }^{18} \mathrm{O}$. B. Christensen and K. W. Jacobsen (to be published).

${ }^{19}$ D. M. Lindsay, Y. Wang, and T. F. George, J. Phys. Chem. 86, 3500 (1987). 